\title{
Chondrocytes treated with different shock wave devices
}

\author{
Angela Notarnicola ${ }^{1}$ \\ Florenzo lannone ${ }^{2}$ \\ Giuseppe Maccagnano ${ }^{1}$ \\ Nuniza Lacarpia² \\ Dorotea Bizzoca ${ }^{2}$ \\ Biagio Moretti ${ }^{1}$
}

1 Orthopaedics Unit, Department of Basic Medical Science, Neuroscience and Sensory Organs, University of Bari, General Hospital, Bari, Italy

2 Interdisciplinary Department of Medicine-Rheumatology Unit, University of Bari, General Hospital, Bari, Italy

\section{Corresponding author:}

Angela Notarnicola

Orthopaedics Unit, Department of Basic Medical Science, Neuroscience and Sensory Organs, University of Bari, General Hospital

Piazza G. Cesare 11

70124 Bari, Italy

E-mail: angelanotarnicola@yahoo.it

\section{Summary}

Background: Shock wave treatment is used for several orthopedic diseases and there are different devices available. Until now, there have been no experimental studies on the effects of these different generators.

Methods: We carried out an experimental study to compare the effects of three focused generators (electro-magnetic, piezoelectric and electro-hydraulic) as well as a radial generator on healthy and osteoarthritis chondrocytes.

Results: By the analysis of our results, we may exclude significant differences between the different generators, even though there is a greater action specificity for electro-magnetic and piezoelectric generators.

Conclusions: The smaller size of the focus of the latter two generators guarantees a greater concentration of energy in the target. The biological effect of the increase of IL-10 and reduction of both $\mathrm{N}$ Cadherin and B-Catenin in chondrocytes in healthy subjects and those affected by osteoarthritis confirms the therapeutic potential of ESWT in cartilage diseases, such as osteoarthritis. In clinical practice it is important to introduce the parameter of total energy. This allows us to standardize the treatment and to manage the variability related to the different types of device and size of the focus.

Level of evidence: Ilb.

KEY WORDS: shock waves, devices, chondrocytes.

\section{Introduction}

In rehabilitation, there are four different methods of physical stimulation techniques: inductive (electromagnetic fields), capacitive (electric fields), faradic (electric current) and mechanical vibration (shock wave, radial wave) ${ }^{1}$. A Shockwave (SW) has an acoustic wave characterized by a quick pressure increase and by a rapid decrease of values below those of the atmosphere, in a few nanoseconds ${ }^{2}$. This physical characteristic determines the focalization of energy in a small area (focus) with the maximum concentration of energy at some $\mathrm{cm}$ of depth of the subcutaneous tissue. The shock wave may be produced by an electro-generator (EI), electromagnetic (EM) and piezoelectric (PE). The SW is responsible for angiogenic effects, modulation of inflammation, as well as proliferative and analgesic effects on the tissue ${ }^{3-5}$. In literature there emerged that the SW induces at the cellular level different metabolic pathways, such as modulation of membrane permeability, the expression of various cytokines and the synthesis of growth factors and nitric oxide. In 2001, there was introduced another type of acoustic wave, called radial wave (RSW), generated by a ballistic system ${ }^{6}$. In the case of the radial wave, the increase in pressure value of the acoustic wave needs a longer time. Furthermore, the maximum energy is found at the interface between skin and transducer and is reduced in a quadratic function related to penetration depth. Until now, there has been a lack of studies on the biological effects. Nevertheless some Authors have reported that the attenuation of the focus may be responsible for reducing the effects of the shock wave ${ }^{7}$. The main indications of shock wave and radial wave in the treatment of musculoskeletal diseases are for tendinopathies, calcific or not, and fracture healing delays $^{8}$. The application in the cartilage diseases, as osteoarthritis, is still preliminary. Only a few clinical and experimental researches have studied the effects of the shock waves and the radial waves on chondrocytes and articular cartilage in the human model9-16. 
The first end-point of this study is to compare the different effects of the shock waves, generated by the three main devices (EI, EM and EP), and the radial waves. The second end-point is to verify the effects of the shock waves and radial waves on the human chondrocyte cell line in normal conditions and in osteo-arthritic disease.

\section{Materials and methods}

Patients ( 9 males and 9 females) with primary severe osteoarthritis (OA) (grade 4 Kellgren and Lawrence radiographic staging) who had undergone joint replacement surgery were recruited into the study and the remaining material was acquired. Osteoarthritic cartilage samples were obtained after knee replacement surgeries. Normal cartilage samples were obtained from traumatic knee lesions. Cartilage was taken from the femoral and tibial sides of knee. Due to the limited size of the sample, we did not perform all experiments on each tissue sample. Patient characteristics are reported in Table I. The research was conducted according to international standards and this study was approved by the local medical ethical committee. The study meets the ethical standards of the Journal ${ }^{17}$. We experienced difficulties in repeating some cell lines, due to contamination of samples after stimulation. For this reason our samples are limited in number. The Cartilage tissue was cut into small pieces (approximately 2 $\mathrm{mm} \times 2 \mathrm{~mm}$ ) and these were then incubated with $0,2 \%$ Collagenase (C0130 Sigma) in DMEM/F12 serum free (1:1; Euroclone ECM 0095L) + Penicillin/Streptomicin (P11-010 Gbco Life Tecnology) and Amphotericin B 1X (P11-001 Gibco Life Tecnology). They were then incubated in $5 \% \mathrm{CO}_{2}$ overnight at $37^{\circ} \mathrm{C}$. The following day, the digested tissue was washed and cultured (25 $\mu \mathrm{g} / \mathrm{ml}$ ascorbic acid was added to the cultured medium). 200,000 chondrocytes by third or fourth passage were stimulated by four shock waves generator systems: electromagnetic device (EM) (Minilith SL1Storz), piezoelectric device (PE) (WellWaves-Wolf), electro hydraulic device (EI) (derma-PACE, Sanuwave) and radial divice (Rad) (SwissDolorcastEMS). The cells were treated with 500 impulses of SW at an energy density of $0.05 \mathrm{~mJ} / \mathrm{mm}^{2}$. On the basis of our previous experiences, we selected a number of pulses and an energy level density efficacy to ensure a biological response ${ }^{4,13}$. Between the generator and the cells we used an ultrasound gel that was compressed in order to guarantee the absence of air. After stimulation, the cells were incubated for 48 hours. Stimulation

Table I. Patients characteristics.

\begin{tabular}{lll}
\hline & FACS analysis & ICC \\
\hline Number & 18 & 18 \\
HD & 8 & 12 \\
OA & 10 & 6 \\
\hline
\end{tabular}

of cells taken from the same patient was performed in the same day. We had the four devices in the same lab. Despite having different device used, we reproduced for each of them the same experimental conditions. The dispensing source was facing upward to ensure a delivery in contact with the plate containing the cells. The depth adjustment of the stimulation was set for all machines as more shallow depth $(<5 \mathrm{~mm})$. All stimulations were carried out over a period of 8 weeks. The Immunocytochemistry (ICC) staining was used to study the expression of $\mathrm{N}$-Cadherin and $\beta$-Catenin. After stimulation, the cells were fixed in formaldehyde $3 \%$ and then permeabilized with Triton X-100. Normal human serum was used to reduce background staining. The cells were incubated overnight with anti-human polyclonal antibodies anti-N-Cadherin (Abcam, 76057) and $\beta$-Catenin (Abcam, 6302) at $4^{\circ} \mathrm{C}$. The next day, secondary biotinylated polyclonal anti-rabbit antibody (E0353 DakoCytomation; 1:200) was used at $4^{\circ}$ C for 30 minutes. After incubation with $3 \%$ hydrogen peroxide for 30 minutes, Vectastain ABC System kit (PK-6200 Vector Laboratories) and DAB Substrate Kit (Vector Laboratories) were used according to the manufacturer's instructions. Mayer's Hematoxylin Solution was used for counterstaining. The data are expressed with a number from 1 to 3 . Three independent observers assigned this visual score (from 1: no staining to 3: high grade staining) by using light microscope (Leica, mod. DM4000B), at 20x magnification.

FACS staining was used to study the expression of IL-10 and N-Cadherin. Approximately 500,000 cells were stained with the following Abs: PE-conjugated IL10 (BD Pharmingen, 559330), N-Cadherin (Abcam, 76057), anti-rat FITC-conjugated IgG (BD, 554020). For the study of IL10 expression, the Cytofix/Cytoperm kit (BD, 554714) was used according to the manufacturer's instructions. All incubations were performed on ice for 30 minutes. The cells were fixed with PFA $1 \%$ and analysed with a FACScan (BD) flow cytometer using CellQuest software. The data are expressed as percentage of positive chondrocytes for expression of IL-10 or $\mathrm{N}$-Cadherin respectively.

The data were expressed as mean +/- standard deviation. Statistical analysis was performed using UMann Whitney tests. SPSS Statistics vers.20 was used. The statistical significance was put for $p<0.05$.

\section{Results}

We studied the expression of IL-10 in normal (HD) and osteoarthritic (OA) cultured chondrocytes after four different stimulations (EM, PE, EI, Rad) by FACS staining (Tab. II). Using different devices, we found significantly higher values in stimulated cells (HD: PE 0.4+/-1.5; EM $0.2+/-0.9$; El 0.1+/- 0.4; RAD 0.08 +/0.3; OA: PE 0.5+/-0.7; EM $0.7+/-0.5)$ compared to the non stimulated cells (HD: $0.1+/-0.5$; OA: $0.01+/-$ $0.4)(p=0.02)$, with a tendency to the highest values in the stimulation with piezoelectric generator in $\mathrm{HD}$ and electromagnetic generator in $O A(p>0.05)$. The 
Table II. Results of the FACS and immunocytochemistry analysis.

\begin{tabular}{|c|c|c|c|c|c|c|c|c|c|}
\hline \multirow[t]{3}{*}{ FACS (\%) } & \multirow{2}{*}{\multicolumn{2}{|c|}{$\begin{array}{l}\text { healthy } \\
\text { IL10 }\end{array}$}} & \multicolumn{3}{|l|}{ OA } & & \multicolumn{3}{|l|}{ OA } \\
\hline & & & \multicolumn{2}{|c|}{ IL10 } & & & \multicolumn{3}{|c|}{ N-Cadherin } \\
\hline & mean & SD & \multicolumn{2}{|c|}{ mean } & SD & & mean & \multicolumn{2}{|l|}{ SD } \\
\hline NS & 0,1 & 0,5 & \multicolumn{2}{|c|}{0,01} & 0,4 & & 41,3 & \multicolumn{2}{|c|}{18,6} \\
\hline PE & 0,4 & 1,5 & \multicolumn{2}{|c|}{0,5} & 0,7 & & 37,4 & \multicolumn{2}{|c|}{19,6} \\
\hline EM & 0,2 & 0,9 & \multicolumn{2}{|l|}{0,7} & 0,5 & & 37,5 & \multicolumn{2}{|c|}{16,9} \\
\hline EI & 0,1 & 0,4 & \multicolumn{2}{|l|}{-} & - & & - & \multicolumn{2}{|c|}{-} \\
\hline RAD & 0,08 & 0,3 & \multicolumn{2}{|l|}{-} & - & & - & \multicolumn{2}{|l|}{ - } \\
\hline \multirow[t]{3}{*}{$\operatorname{ICC}(1-3)$} & healthy & & \multicolumn{3}{|l|}{ OA } & \multicolumn{2}{|c|}{ healthy } & \multicolumn{2}{|l|}{ OA } \\
\hline & \multicolumn{2}{|l|}{$\beta$-Catenin } & \multicolumn{2}{|c|}{$\beta$-Catenin } & & \multicolumn{2}{|c|}{ N-Cadherin } & \multicolumn{2}{|c|}{$\mathrm{N}$-Cadherin } \\
\hline & mean & SD & mean & SD & & mean & SD & mean & SD \\
\hline NS & 2,2 & 0,3 & 2,5 & 0,1 & & 2,1 & 0,4 & 2,4 & 0,2 \\
\hline PE & 1,6 & 0,7 & 1,5 & 0,6 & & 1,3 & 0,6 & 1,7 & 0,5 \\
\hline EM & 1,3 & 0,2 & 1,4 & 0,3 & & 1,2 & 0,3 & 1,3 & 1,5 \\
\hline EI & 1,3 & 0,7 & 1,5 & 0,4 & & 1,5 & 0,6 & 1,6 & 1,6 \\
\hline RAD & 1,5 & 0,8 & 1,4 & 0,7 & & 1,5 & 0,7 & 1,3 & 1,5 \\
\hline
\end{tabular}

data did not show any difference in the expression of IL-10 in healthy (HD) compared to osteoarthritis (OA) chondrocytes after different shock waves stimulation. We studied the expression of $\mathrm{N}$-Cadherin in osteoarthritic (OA) cultured chondrocytes after different stimulations (EM, PE) by FACS staining. The value of non-stimulated cells (OA: $41.3+/-18.6)$ was statistically higher than treated cells (OA: PE 37.4 +/- 19.6; EM $37.5+/-16.9)(p=0.04)$. As regards the PE and EM stimulated cells, there was a tendency towards a decrease in values ( $p>0.05)$.

Then we evaluated the expression of $\beta$-Catenin in HD and $O A$ cultured chondrocytes after the same stimulations by ICC staining. The cells not stimulated with SW (HD: 2.2 +/- 0.3; OA: 2.5 +/- 0.1) showed higher values than those stimulated (HD: PE $1.6+/-0.7$; EM $1.3+/-0.2$; OA: PE $1.5+/-0.6$; EM $1.4+/-0.3$ ) $(p=0.02)$. We found a trend towards a decrease in values in the cells stimulated with EM generator $(p>0.05)$. Furthermore, we evaluated the expression of $\mathrm{N}$-Cadherin in $\mathrm{HD}$ and $\mathrm{OA}$ cultured chondrocytes after the same stimulations by ICC staining. As regards the non-stimulated cells (HD: 2.1 +/- 0.4; OA: $2.4+/-0.2)$, we revealed statistically greater values (HD: PE 1.3 +/- 0.6; EM 1.2 +/- 0.2; OA: PE 1.7 +/0.5 ; EM 1.3+/- 1.5) $(p=0.01)$. We found a trend towards a decrease in values in the cells stimulated using EM generator ( $p>0.05)$.

\section{Discussion}

We conducted this study in order to respond to the controversy in the scientific community as regards whether or not there are differences between the different acoustic wave generators. The results, despite the limited sample and the difficulty to increase the number of cases, thereby hindering reproducibility, allows us to make interesting hypotheses regarding previous knowledge reported in the literature. Until now, there has not been conducted an experimental or clinical study which compares directly all of the different generators. In 2006, Martini et al., in an experimental study compared the effects of electromagnetic and electrohydraulic devices. They found that the electromagnetic device induces fewer cytodestructive effects and more proliferation stimulation on osteoclast cells ${ }^{18}$. Our results do not support this first hypothesis, because we verified that different types of generators caused similar effects in cell biological response. Furthermore, the tendency to increased action with $\mathrm{EM}$ and $\mathrm{PE}$ devices, may be interpreted in relation to the smaller size of the focus of these generators, with consequent major focalization of the treatment and major concentration of the dissipated energy.

The second aim of the work was to analyze the effects of the SW on the cartilage. In the animal model it was verified that the application of SW does not induce pathological changes in the articular cartilage $e^{9,11,13,19,20}$. Furthermore, SW improves joint function, reduces inflammatory cytokines which are responsible for osteoarthritic degeneration and enhances the recovery effects ${ }^{13}$. Recently, three clinical studies support the potential therapeutic utility of SW in the treatment of $\mathrm{OA}^{21-23}$. IL-10 inhibits the synthesis of different pro-inflammatory cytokines (IFN- $\mathrm{Y}, \mathrm{IL}-2$, IL-3, TNF-a and GM-CSF) which are hyper-expressed in arthritic conditions. The overexpression of $\mathrm{N}$-Cadherin and $\beta$-Catenin is associated with degeneration diseases ${ }^{24}$. Our results show a trend towards an increased expression of IL-10 and a decreased 
expression of $\mathrm{N}$-Cadherin and $\beta$-Catenin after stimulation with different devices. Our data do not allow to confirm that the extracorporeal shockwave therapy is effective in the treatment of osteoarthritis. Further experimental and clinical studies are needed to validate the clinical application of the SW in the treatment of $\mathrm{OA}$ and to assign levels of evidence of effectiveness. In conclusion, by observation of the results of our study, we may support an overlap of effects of different shock waves generators. The introduction of the total energy parameter with respect to the EDF (Energy Flux Density) parameter only, currently used to quantify the energy delivered during ESW treatment, may allow us to overcome the possible differences determined by the focal range of different sizes of each generator. This will be useful in order to conform the treatment protocols and the results of the therapies provided with each generator. Also the stimulation with radial waves would appear to be able to determine the biological and therapeutic effects, notwithstanding the physical differences between the radial wave and the shock wave. This is consistent with the new recent experiences on the biological effects and the therapeutic potential of different mechanical stimulations, from mechano-transduction to vibration ${ }^{9,25,26}$. The applications of shock waves in the treatment of cartilage and osteoarthritis will represent the next frontier, after wide spread application of this therapy in tendon pathologies ${ }^{27-29}$. Further clinical and in vivo studies are needed to test the effects on humans and define specific treatment protocols.

\section{Conflict of interest}

The Author has no financial or personal relationships with other people or organizations that could inappropriately influence their work.

\section{References}

1. Moretti B. Biofisica ortopedica e traumatologia nella pratica clinica. CIC Edizioni Internazionali. 2015.

2. Staudenraus J. In vivo Strasswllenmessung. In Chaussy (Hrsg). Die Stosswelle in Forschung und Kinik. Attempto Verlag. 1995;S21S26.

3. Buselli P, Coco V, Notarnicola A, et al. Shock waves in the treatment of post-traumatic myositis ossificans. Ultrasound Med Biol. 2010;36(3):397-409.

4. Iannone F, Moretti B, Notarnicola A, et al.. Extracorporeal shock waves increase interleukin-10 expression by human osteoarthritic and healthy osteoblasts in vitro. Clin Exp Rheumatol. 2009;27(5):794-799.

5. Notarnicola A, Maccagnano G, Tafuri S, Fiore A, Pesce V, Moretti B. Comparison of shock wave therapy and nutraceutical composed of Echinacea angustifolia, alpha lipoic acid, conjugated linoleic acid and quercetin (perinerv) in patients with carpal tunnel syndrome. Int J Immunopathol Pharmacol. 2015;28(2):256-262.

6. Notarnicola A, Tamma R, Moretti L, et al. Effects of radial shock waves therapy on osteoblasts activities. Musculoskelet Surg. 2012;96(3);183-189.
7. Cleveland RO, Chitnis PV, McClure SR. Acoustic field of a ballistic shock wave therapy device. Ultrasound Med Biol. 2007;33(8):13271335.

8. Romeo P, Lavanga V, Pagani D, Sansone V. Extracorporeal shock wave therapy in musculoskeletal disorders: a review. Med Princ Pract. 2014;23(1):7-13.

9. Benson BM, Byron CR, Pondenis $\mathrm{H}$, Stewart AA. The effects of radial shock waves on the metabolism of equine cartilage explants in vitro. 2007;55(1):40-44.

10. Byron CR, Benson BM, Stewart AA, Stewart MC. Effects of radial shock waves on membrane permeability and viability of chondrocytes and structure of articular cartilage in equine cartilage explants. Am J Vet Res. 2005;66(10):1757-1763.

11. Dorotka R, Kubista B, Schatz KD. Trieb: Effects of extracorporeal shock waves on human articular chondrocytes and ovine bone marrow stromal cells in vitro. Arch Orthop Trauma Surg. 2003;123(7);345-348.

12. Mayer-Wagner S, Ernst J, Maier M, et al. The effect of high-energy extracorporeal shock waves on hyaline cartilage of adult rats in vivo. J Orthop Res. 2010;28(8):1050-1056.

13. Moretti B, lannone F, Notarnicola A, et al. Extracorporeal shock waves down-regulate the expression of interleukin-10 and tumor necrosis factor-alpha in osteoarthritic chondrocytes. BMC Musculoskelet Disord. 2008;31:9;16.

14. Moretti B, Notarnicola A, Moretti L, Giordano P, Patella V. A volleyball player with bilateral knee osteochondritis dissecans treated with extracorporeal shock wave therapy. Chir Organi Mov. 2009;93(1):37-41.

15. Renz H, Rupp S. Effects of shock waves on chondrocytes and their relevance in clinical practice: Arch Orthop Trauma Surg. 2009;129(5):641-647.

16. Zhao Z, Ji H, Jing R, et al. Extracorporeal shock-wave therapy reduces progression of knee osteoarthritis in rabbits by reducing nitric oxide level and chondrocyte apoptosis. Arch Orthop Trauma Surg. 2012;132(11):1547-1553.

17. Padulo J, Oliva F, Frizziero A, Maffulli N. Muscles, Ligaments and Tendons Journal Basic principles and recommendations in clinical and field Science Research: 2016 Update. MLTJ. 2016;6(1):1-5.

18. Martini L, Giavaresi G, Fini M, Borsari V, Torricelli P, Giardino R. Early effects of extracorporeal shock wave treatment on osteoblast-like cells: a comparative study between electromagnetic and electrohydraulic devices. J Trauma. 2006;61(5): 1198-1206.

19. Murata R, Nakagawa K, Ohtori S, et al. The effects of radial shock waves on gene transfer in rabbit chondrocytes in vitro. Osteoarthritis Cartilage. 2007;15(11);1275-1282.

20. Zhao Z, Jing R, Shi Z, Zhao B, Ai Q, Xing G. Efficacy of extracorporeal shockwave therapy for knee osteoarthritis: a randomized controlled trial. J Surg Res. 2013;185(2):661-666.

21. Chen TW, Lin CW, Lee CL, et al.The efficacy of shock wave therapy in patients with knee osteoarthritis and popliteal cyamella. Kaohsiung J Med Sci. 2014;30(7):362-370.

22. Razumov AN, Puriga AO, Yurova OV. The long-term results of the application of the combined rehabilitative treatment in the patients presenting with knee. Vopr Kurortol Fizioter Lech Fiz Kult. 2015;92(6):42-44.

23. Xu HG, Zheng Q, Song JX, et al. Intermittent cyclic mechanical tension promotes endplate cartilage degeneration via canonical Wnt signaling pathway and E-cadherin/ $\beta$-catenin complex cross-talk. Osteoarthritis Cartilage. 2016;24(1):158168.

24. Berschin G, Sommer B, Behrens A, Sommer HM. Whole Body Vibration Exercise Protocol versus a Standard Exercise Protocol after ACL Reconstruction: A Clinical Randomized Controlled Trial with Short Term Follow-Up. J Sports Sci Med. 2014;1,13(3):580-589.

25. Dunn SL, Olmedo ML. Mechanotransduction. Relevance to 
Physical Therapist Practice-Understanding Our Ability to Affect Genetic Expression Through Mechanical Forces. Phys Ther. 2015;23.

26. Frairia R, Berta L. Biological effects of extracorporeal shock waves on fibroblasts. A review. Muscles Ligaments Tendons J. 2011;1(4):138-147.

27. Notarnicola A, Moretti B. The biological effects of extracorporeal shock wave therapy (eswt) on tendon tissue. Muscles Ligaments Tendons J. 2012;2(1):33-37.
28. Manganotti $P$, Amelio E, Guerra C. Shock wave over hand muscles: a neurophysiological study on peripheral conduction nerves in normal subjects. Muscles Ligaments Tendons $\mathrm{J}$. 2012;2(2):104-107.

29. Visco V, Vulpiani MC, Torrisi MR, Ferretti A, Pavan A, Vetrano $M$. Experimental studies on the biological effects of extracorporeal shock wave therapy on tendonmodels. A review of the literature. Muscles Ligaments Tendons J. 2014;17;4(3):357361. 